\title{
Disk Diffusion Susceptibility Testing for the Rapid Detection of Fluconazole Resistance in Candida Isolates
}

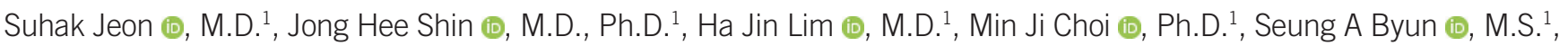

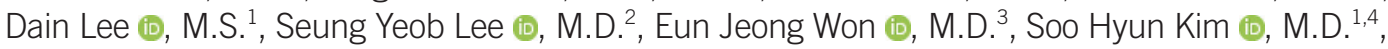
and Myung Geun Shin $\mathbb{0}$, M.D. ${ }^{1}$

${ }^{1}$ Department of Laboratory Medicine, Chonnam National University Medical School and Chonnam National University Hospital, Gwangju, Korea; ${ }^{2}$ Department of Laboratory Medicine, Jeonbuk National University Medical School and Jeonbuk National University Hospital, Jeonju, Korea; Departments of ${ }^{3}$ Parasitology and Tropical Medicine and ${ }^{4}$ Microbiology and Laboratory Medicine, Chonnam National University Medical School, Hwasun, Korea

Background: Given the increased fluconazole resistance (FR) among Candida isolates, we assessed the suitability of disk diffusion susceptibility testing (DDT) for the early detection of FR using well-characterized Candida isolates.

Methods: In total, 188 Candida isolates, including 66 C. albicans (seven Erg11 mutants), 69 C. glabrata (33 Pdr1 mutants), 29 C. parapsilosis (15 Erg11 mutants), and 24 C. tropicalis (eight Erg11 mutants) isolates, were tested in this study. FR was assessed using DDT according to the standard CLSI M44-ED3 method, except that two cell suspensions, McFarland 0.5 (standard inoculum) and 2.5 (large inoculum), were used, and the inhibition zones were read at 2-hour intervals from 10 hours to 24 hours.

Results: DDT results for the standard inoculum were readable after 14 hours (C. albicans, C. glabrata, and C. tropicalis) and 20 hours (C. parapsilosis) for $>95 \%$ of the isolates, whereas the results for the large inoculum were readable after 12 hours (C. glabrata and C. tropicalis), 14 hours (C. albicans), and 16 hours (C. parapsilosis) for $>95 \%$ of the isolates. Compared with the results produced using the CLSI M27-ED4 broth microdilution method, the first readable results from the DDT method for each isolate exhibited an agreement of $97.0 \%, 98.6 \%, 72.4 \%$, and $91.7 \%$ for the standard inoculum and $100 \%$, 98.6\%, 96.6\%, and 95.8\% for the large inoculum for C. albicans, C. glabrata, C. parapsilosis, and C. tropicalis, respectively.

Conclusions: DDT using large inoculum may detect FR rapidly and reliably in the four most common Candida species.

Key Words: Candida, Fluconazole, Resistance, Disk diffusion, CLSI, Antifungal susceptibility testing
Received: November 6, 2020

Revision received: December 28, 2020

Accepted: May 20, 2021

\section{Corresponding author:}

Jong Hee Shin, M.D., Ph.D.

Department of Laboratory Medicine, Chonnam National University Medical School, 42 Jebong-ro, Dong-gu, Gwangju 61469, Korea

Tel: +82-62-220-5342

Fax +82-62-224-2518

E-mail: shinjh@chonnam.ac.kr

\section{cc) (1) (8)}

(c) Korean Society for Laboratory Medicine

This is an Open Access article distributed under the terms of the Creative Commons Attribution Non-Commercial License (https://creativecommons.org/licenses/by-nc/4.0) which permits unrestricted non-commercial use, distribution, and reproduction in any medium, provided the original work is properly cited.

\section{INTRODUCTION}

Candida is an important fungal pathogen that cause invasive candidiasis, leading to significant morbidity and mortality worldwide [1, 2]. Most cases of invasive candidiasis are attributable to C. albicans, C. glabrata, C. parapsilosis, and C. tropicalis [3]. Fluconazole is among the most widely prescribed antifungal agents and is an important first-line treatment for invasive candidiasis owing to its potent efficacy, low cost, and broad-spectrum activity against various Candida species [4, 5]. However, 
the widespread use of azole antifungals has resulted in the emergence of Candida isolates with acquired fluconazole resistance (FR), resulting in recent revisions of the clinical breakpoints (CBPs) because of the wide array of molecular mechanisms underlying resistance $[3,6,7]$. Therefore, fluconazole susceptibility testing is recommended for all bloodstream infections and clinically relevant Candida isolates, as it is useful in optimizing the treatment of invasive candidiasis [5].

Disk diffusion testing (DDT), one of the oldest approaches to susceptibility testing, is still widely used in clinical laboratories because of its low cost and technical simplicity [8]. DDT has recently been used for the direct testing of bacterial or fungal susceptibility in positive blood cultures [9-11]. Methods for shortening the incubation times for DDT have recently been reported for several common bacterial pathogens, enabling faster reporting of antimicrobial susceptibility test results from positive blood cultures $[9,12]$. However, methods for shortening the incubation times for DDT for the detection of FR Candida isolates are less reported. Further, fluconazole susceptibility testing of Candida isolates using the DDT method has not been thoroughly evaluated, and previous studies using comparison with the CLSI M27 broth microdilution (BMD) reference method relied on tentative interpretive criteria that were applied to all Candida species $[8,13,14]$.

We evaluated the performance of the DDT for the early detection of FR in four common Candida species using a panel of wellcharacterized Candida isolates from a Korean collection composed of FR isolates with various molecularly defined azole resistance mechanisms as well as a panel of wild-type isolates without detectable azole resistance mechanisms. Categorical fluconazole susceptibility results obtained using the DDT were compared with those obtained using the CLSI M27-ED4 BMD reference method, using revised species-specific CBPs, as defined in the CLSI M60-ED2 document [15, 16]. To our knowledge, ours is the first study to evaluate the performance of DDT for the early detection of FR among Candida isolates using a panel of Candida isolates with known and unknown resistance mechanisms.

\section{MATERIALS AND METHODS}

\section{Candida isolates and azole resistance mechanisms}

In total, 188 clinical isolates (186 from blood, one from urine, and one from pus) of four common Candida species from a Korean collection were tested. The collection included $66 \mathrm{C}$. albicans, 69 C. glabrata, 29 C. parapsilosis, and 24 C. tropicalis iso- lates that were submitted to Chonnam National University Hospital, Gwangju, Korea, from several Korean hospitals during a nationwide surveillance (2003-2019). All isolates were stored at $-70^{\circ} \mathrm{C}$ in trypticase soy broth supplemented with $15 \%$ glycerol until testing and were passaged twice on potato dextrose agar at $35^{\circ} \mathrm{C}$ prior to testing in 2019. The isolates were identified to the species level using biochemical tests, matrix-assisted laser-desorption ionization-time of flight mass spectrometry, and/or molecular methods [17-19]. FR of all isolates was determined using the CLSI M27 BMD method [15]. For all C. albicans and C. parapsilosis isolates, ERG11 and three transcription factor genes, i.e., TAC1, MRR1, and UPC2, were sequenced [7, 20]. For all C. glabrata isolates, PDR1 was sequenced [21]. For all $C$. tropicalis isolates, CDR1, MDR1, and ERG11 expression was quantified, and ERG11 and UPC2 were sequenced [22]. The full study panel consisted of $C$. albicans (59 wild-type fluconazole-susceptible [FS] isolates and seven FR isolates harboring the K143R or G464S variant in Erg11p), C. glabrata (36 wild-type FS dose-dependent [F-SDD] isolates and $33 \mathrm{FR}$ isolates harboring a Pdr1 variant), C. parapsilosis (14 wild-type FS isolates and $15 \mathrm{FR}$ isolates harboring the $\mathrm{Y} 132 \mathrm{~F}$ variant in Erg11p), and $\mathrm{C}$. tropicalis (16 wild-type $\mathrm{FS}$ isolates, seven FR isolates overexpressing ERG11, and one FR isolate harboring the Y132F variant in Erg11p) isolates (Table 1). This study was approved by the Institutional Review Board of Chonnam National University Hospital, Gwangju, Korea (IRB CNUH-2018-283) which waived the requirement for informed consent.

\section{Antifungal susceptibility testing}

For all Candida isolates, the fluconazole minimal inhibitory concentration (MIC) was re-determined by the CLSI M27 BMD method after 24 hours of incubation [15]. Fluconazole MICs were categorized using species-specific CBPs provided in CLSI M60-ED2 document, as follows: MIC $\leq 2 \mu \mathrm{g} / \mathrm{mL}$ (FS), $4 \mu \mathrm{g} / \mathrm{mL}$ (F-SDD), and $\geq 8 \mu \mathrm{g} / \mathrm{mL}$ (FR) for $C$. albicans, C. parapsilosis, and C. tropicalis, and MIC $\leq 32 \mu \mathrm{g} / \mathrm{mL}$ (F-SDD) and $\geq 64 \mu \mathrm{g} / \mathrm{mL}$ (FR) for $C$. glabrata [16]. DDT was performed according to the CLSI M44ED3 method using a 25- $\mu$ f fluconazole disk (Oxoid, Basingstoke, UK) placed on Mueller-Hinton agar supplemented with $2 \%$ dextrose and $0.5 \mu \mathrm{g} / \mathrm{mL}$ methylene blue [23]. Inoculation was performed using cell suspensions of McFarland 0.5 (standard inoculum) and 2.5 (large inoculum), and results were read at 2-hour intervals from 10 hours to 24 hours. Average cell numbers (ranges) of each Candida species were 2.3-6.1 $\times 10^{6}(1.5-$ $\left.8.3 \times 10^{6}\right)$ colony-forming units (CFU)/mL for the standard inoculum and $1.3-3.1 \times 10^{7}\left(0.9-3.9 \times 10^{7}\right) \mathrm{CFU} / \mathrm{mL}$ for the large in- 
Table 1. Categorical agreement between fluconazole susceptibility results determined by the BMD and DDT for 188 Candida isolates with known and unknown molecular mechanisms

\begin{tabular}{|c|c|c|c|c|c|c|c|c|c|c|}
\hline \multirow[t]{2}{*}{ Species } & \multirow{2}{*}{$\begin{array}{l}\text { Fluconazole } \\
\text { category by } \\
\text { CLSI M27* }\end{array}$} & \multirow[t]{2}{*}{ Defined resistance mechanism } & \multirow{2}{*}{$\begin{array}{l}\mathrm{N} \text { of } \\
\text { isolates }\end{array}$} & \multicolumn{3}{|c|}{$\begin{array}{l}\mathrm{N} \text { of isolates with DDT result for } \\
\text { fluconazole }{ }^{\dagger}\end{array}$} & \multirow[t]{2}{*}{$\% \mathrm{CA}$} & \multicolumn{3}{|c|}{$\begin{array}{l}\mathrm{N}(\%) \text { of isolates with } \\
\text { discrepancies }^{\dagger}\end{array}$} \\
\hline & & & & FR & F-SDD & FS & & VME & ME & Minor \\
\hline \multirow[t]{3}{*}{ C. albicans } & FR & ERG11, K143R or G464S variant & 7 & 5 & 0 & 2 & & & & \\
\hline & FS & $\begin{array}{l}\text { No variants (ERG11, TAC1, MRR1, and } \\
\text { UPC2) defined }\end{array}$ & 59 & 0 & 0 & 59 & & & & \\
\hline & Total & & 66 & 5 & 0 & 61 & 97.0 & $2(3.0)$ & $0(0.0)$ & $0(0.0)$ \\
\hline \multirow[t]{3}{*}{ C. glabrata } & FR & $P D R 1$ variants & 33 & 33 & 0 & 0 & & & & \\
\hline & F-SDD & № PDR1 variants & 36 & 1 & 35 & 0 & & & & \\
\hline & Total & & 69 & 34 & 35 & 0 & 98.6 & $0(0.0)$ & $0(0.0)$ & $1(1.4)$ \\
\hline \multirow[t]{3}{*}{ C. parapsilosis } & $\mathrm{FR}$ & ERG11, Y132F variant & 15 & 12 & 2 & 1 & & & & \\
\hline & FS & $\begin{array}{l}\text { No variants (ERG11, TAC1, MRR1, and } \\
\text { UPC2) defined }\end{array}$ & 14 & 0 & 0 & 14 & & & & \\
\hline & Total & & 29 & 12 & 2 & 15 & 89.7 & $1(3.4)$ & $0(0.0)$ & $2(6.9)$ \\
\hline \multirow[t]{3}{*}{ C. tropicalis } & FR & ERG11 overexpression or $\mathrm{Y} 132 \mathrm{~F}$ variant & 8 & 7 & 1 & 0 & & & & \\
\hline & FS & No variants (ERG11 and UPC2) defined & 16 & 0 & 0 & 16 & & & & \\
\hline & Total & & 24 & 7 & 1 & 16 & 95.8 & $0(0.0)$ & $0(0.0)$ & $1(4.2)$ \\
\hline \multirow[t]{3}{*}{ Total } & FR & $\begin{array}{l}\text { Presence of defined azole resistance } \\
\text { mechanisms }\end{array}$ & 63 & 57 & 3 & 3 & & & & \\
\hline & FS/F-SDD & No azole variant defined & 125 & 1 & 35 & 89 & & & & \\
\hline & Total & & 188 & 58 & 38 & 92 & 96.3 & $3(1.6)$ & $0(0.0)$ & $4(2.1)$ \\
\hline
\end{tabular}

*Fluconazole susceptibility testing was performed according to the CLSI M27 BMD-ED4 method [15]. Categorical results were determined using the CLSI M60-ED2 method [16]. ' ${ }^{\dagger}$ luconazole susceptibility testing was performed according to the CLSI M44-ED3 method [23]. Categorical results were determined using the CLSI M60-ED2 method [16].

Abbreviations: BMD, broth microdilution; DDT, disk diffusion testing; CA, categorical agreement; FR, fluconazole resistant; FS, fluconazole susceptible; FSDD, fluconazole-susceptible dose-dependent; ME, major error; Minor, minor error; VME, very major error.

oculum (Table 2).

The diameter of the growth inhibition zone was measured across the area in which growth was prominently reduced. The interpretive criteria for DDT are as follows: an inhibition zone diameter $\geq 17 \mathrm{~mm}$ indicates FS, a diameter of $14-16 \mathrm{~mm}$ indicates F-SDD, and a diameter $\leq 13 \mathrm{~mm}$ indicates FR for $C$. albicans, C. parapsilosis, and C. tropicalis. For C. glabrata, an inhibition zone diameter $\geq 15 \mathrm{~mm}$ indicates F-SDD, and a diameter $\leq 14 \mathrm{~mm}$ indicates FR [16]. In each test, two reference isolates (C. parapsilosis American Type Culture Collection (ATCC) 22019 and C. krusei ATCC 6258) were included as controls.

\section{Data and statistical analyses}

Categorical agreement (CA) was determined as the percentage of isolates classified into the same FS or FR categories using both DDT and the CLSI M27 BMD method. Errors were classified as a very major error (VME) when an isolate classified as FR by the reference BMD method was categorized as FS by DDT, a
Table 2. Number of yeast cells $/ \mathrm{mL}$ of the 0.5 and $2.5 \mathrm{McF}$ arland suspensions of four common Candida species

\begin{tabular}{|c|c|c|c|}
\hline \multirow{2}{*}{ Species } & \multirow{2}{*}{ Inoculum* } & \multicolumn{2}{|c|}{ Number of cells $\left(\times 10^{6} \mathrm{CFU} / \mathrm{mL}\right)$} \\
\hline & & Mean \pm SD & Range \\
\hline \multirow[t]{2}{*}{ C. albicans } & Standard & $5.2 \pm 0.9$ & $3.8-6.9$ \\
\hline & Large & $26.5 \pm 4.3$ & $20.3-34.7$ \\
\hline \multirow[t]{2}{*}{ C. glabrata } & Standard & $6.1 \pm 1.1$ & $4.3-8.3$ \\
\hline & Large & $31.2 \pm 4.8$ & $23.3-39.0$ \\
\hline \multirow[t]{2}{*}{ C. parapsilosis } & Standard & $3.4 \pm 0.6$ & $2.5-4.2$ \\
\hline & Large & $17.0 \pm 2.5$ & 13.1-20.7 \\
\hline \multirow[t]{2}{*}{ C. tropicalis } & Standard & $2.3 \pm 1.0$ & $1.5-4.7$ \\
\hline & Large & $13.3 \pm 5.6$ & $8.5-26.8$ \\
\hline \multirow[t]{2}{*}{ Total } & Standard & $4.3 \pm 1.8$ & $1.5-8.3$ \\
\hline & Large & $22.0 \pm 8.4$ & $8.5-39.0$ \\
\hline
\end{tabular}

${ }^{*}$ Colony counting was performed with the use of two cell suspensions (McFarland 0.5, standard inoculum; and 2.5, large inoculum). ${ }^{\dagger}$ Quantification was performed using a Neubauer chamber. Mean values of 10 isolates of each species are presented. 
Table 3. Categorical agreement between fluconazole susceptibility results determined by DDT using two inocula and the BMD method after each incubation time for the readable Candida isolates*

\begin{tabular}{|c|c|c|c|c|c|c|c|c|c|c|c|}
\hline \multirow{3}{*}{$\begin{array}{l}\text { Species } \\
\text { (N tested) }\end{array}$} & \multirow{3}{*}{$\begin{array}{l}\text { Incubation } \\
\text { time } \\
\text { (hr) }\end{array}$} & \multicolumn{5}{|c|}{ DDT using a standard inoculum (McFarland 0.5) } & \multicolumn{5}{|c|}{ DDT using a large inoculum (McFarland 2.5) } \\
\hline & & \multirow{2}{*}{$\begin{array}{l}\text { Readable } \\
\mathrm{N}(\%)\end{array}$} & \multirow{2}{*}{$\% \mathrm{CA}$} & \multicolumn{3}{|c|}{$\mathrm{N}(\%)$ of isolates with discrepancies } & \multirow{2}{*}{$\begin{array}{l}\text { Readable } \\
\mathrm{N}(\%)\end{array}$} & \multirow{2}{*}{$\%$ CA } & \multicolumn{3}{|c|}{$\mathrm{N}(\%)$ of isolates with discrepancies } \\
\hline & & & & VME & ME & Minor & & & VME & ME & Minor \\
\hline \multirow{5}{*}{$\begin{array}{l}\text { C. albicans } \\
\text { (66) }\end{array}$} & 12 & $38(57.6)$ & 100.0 & 0 & 0 & 0 & $55(83.3)^{\dagger}$ & 96.4 & $1(1.8)$ & 0 & $1(1.8)$ \\
\hline & 14 & $63(95.5)$ & 96.8 & $2(3.2)$ & 0 & 0 & $63(95.5)^{\dagger}$ & 96.8 & $1(1.6)$ & 0 & $1(1.6)$ \\
\hline & 20 & $64(97.0)$ & 96.9 & $2(3.1)$ & 0 & 0 & $66(100.0)$ & 95.5 & $1(1.5)$ & 0 & $2(3.0)$ \\
\hline & 22 & $66(100.0)$ & 97.0 & $2(3.0)$ & 0 & 0 & $66(100.0)$ & 97.0 & $1(1.5)$ & 0 & $1(1.5)$ \\
\hline & 24 & $66(100.0)$ & 97.0 & $2(3.0)$ & 0 & 0 & $66(100.0)$ & 97.0 & $1(1.5)$ & 0 & $1(1.5)$ \\
\hline \multirow{5}{*}{$\begin{array}{l}\text { C. glabrata } \\
\text { (69) }\end{array}$} & 10 & $46(66.7)$ & 100.0 & 0 & 0 & 0 & $60(87.0)^{\dagger}$ & 100.0 & 0 & 0 & 0 \\
\hline & 18 & $69(100.0)$ & 98.6 & 0 & 0 & $1(1.4)$ & $69(100.0)$ & 98.6 & 0 & 0 & $1(1.4)$ \\
\hline & 20 & $69(100.0)$ & 98.6 & 0 & 0 & $1(1.4)$ & $69(100.0)$ & 98.6 & 0 & 0 & $1(1.4)$ \\
\hline & 22 & $69(100.0)$ & 98.6 & 0 & 0 & $1(1.4)$ & $69(100.0)$ & 97.1 & 0 & 0 & $2(2.9)$ \\
\hline & 24 & $69(100.0)$ & 98.6 & 0 & 0 & $1(1.4)$ & $69(100.0)$ & 94.2 & 0 & 0 & $4(5.8)$ \\
\hline \multirow{6}{*}{$\begin{array}{l}\text { C. parapsilosis } \\
\text { (29) }\end{array}$} & 10 & $0(0.0)$ & - & 0 & 0 & 0 & $2(6.9)$ & 100.0 & 0 & 0 & 0 \\
\hline & 12 & $1(3.4)$ & 100.0 & 0 & 0 & 0 & $24(82.8)^{\dagger}$ & 95.8 & 0 & 0 & $1(4.2)$ \\
\hline & 14 & $19(65.5)$ & 68.4 & $1(5.3)$ & 0 & $5(26.3)$ & $26(89.7)^{\dagger}$ & $92.3^{\dagger}$ & 0 & 0 & $2(7.7)$ \\
\hline & 16 & $22(75.9)$ & 90.9 & 0 & 0 & $2(9.1)$ & $29(100.0)^{\dagger}$ & 93.1 & 0 & 0 & $2(6.9)$ \\
\hline & 18 & 25 (86.2) & 92.0 & 0 & 0 & $2(8.0)$ & $29(100.0)^{\dagger}$ & 93.1 & 0 & 0 & $2(6.9)$ \\
\hline & 20 & $29(100.0)$ & 89.7 & $2(6.9)$ & 0 & $1(3.4)$ & $29(100.0)$ & 93.1 & 0 & 0 & $2(6.9)$ \\
\hline \multirow{4}{*}{$\begin{array}{l}\text { C. tropicalis } \\
(24)\end{array}$} & 18 & $24(100.0)$ & 95.8 & 1 (4.2) & 0 & 0 & $24(100.0)$ & 95.8 & 1 (4.2) & 0 & 0 \\
\hline & 20 & $24(100.0)$ & 95.8 & 0 & 0 & 1 (4.2) & $24(100.0)$ & 95.8 & 1 (4.2) & 0 & 0 \\
\hline & 22 & $24(100.0)$ & 95.8 & 0 & 0 & $1(4.2)$ & $24(100.0)$ & 95.8 & 0 & 0 & $1(4.2)$ \\
\hline & 24 & $24(100.0)$ & 95.8 & 0 & 0 & $1(4.2)$ & $24(100.0)$ & 95.8 & 0 & 0 & $1(4.2)$ \\
\hline \multirow[t]{8}{*}{ Total (188) } & 10 & $66(35.1)$ & 98.5 & 0 & 0 & $1(1.5)$ & $110(58.5)^{\dagger}$ & 99.1 & $1(0.9)$ & 0 & 0 \\
\hline & 12 & $120(63.8)$ & 98.3 & $1(0.8)$ & 0 & $1(0.8)$ & $171(91.0)^{\dagger}$ & 95.9 & $2(1.2)$ & 0 & $5(2.9)$ \\
\hline & 14 & $173(92.0)$ & 93.6 & $4(2.3)$ & 0 & $7(4.0)$ & $182(96.8)^{\dagger}$ & 96.2 & $2(1.1)$ & 0 & $5(2.7)$ \\
\hline & 16 & $178(94.7)$ & 95.5 & $3(1.7)$ & 0 & $5(2.8)$ & $188(100.0)^{\dagger}$ & 96.3 & $2(1.1)$ & 0 & $5(2.7)$ \\
\hline & 18 & 181 (96.3) & 96.1 & $3(1.7)$ & 0 & $4(2.2)$ & $188(100.0)^{\dagger}$ & 96.3 & $3(1.6)$ & 0 & $4(2.1)$ \\
\hline & 20 & $186(98.9)$ & 96.2 & $4(2.2)$ & 0 & $3(1.6)$ & $188(100.0)$ & 96.3 & $2(1.1)$ & 0 & $5(2.7)$ \\
\hline & 22 & $188(100.0)$ & 96.3 & $4(2.1)$ & 0 & $3(1.6)$ & $188(100.0)$ & 96.3 & $1(0.5)$ & 0 & $6(3.2)$ \\
\hline & 24 & $188(100.0)$ & 96.3 & $3(1.6)$ & 0 & $4(2.1)$ & $188(100.0)$ & 95.7 & $1(0.5)$ & 0 & $7(3.7)$ \\
\hline
\end{tabular}

*BMD method was conducted strictly according to CLSI M27-ED4 document (24-hour incubation), but DDT was modified with the use of two cell suspensions (McFarland 0.5, standard inoculum; and 2.5, large inoculum), and inhibition zones were read at 2-hour intervals from 10 hours to 24 hours [15]. ${ }^{\dagger} P<0.05$, significant difference between the standard and large inoculums within a given category (i.e., readable N; \% CA) and a given Candida species. Abbreviations: BMD, broth microdilution; CA, categorical agreement; DDT, disk diffusion testing; ME, major error; Minor, minor error; VME, very major error. 
major error (ME) when an isolate classified as FS by the BMD method was classified as FR by DDT, or a minor error for all other discordances. The chi-square or Fisher's exact test was used to compare CA rates, and Student's $t$-test was used to compare the test time. Statistical analysis was performed using $\mathrm{R}$ version 4.0.3 (The R Project for Statistical Computing, Vienna, Austria), and significance was defined as $P<0.05$.

\section{RESULTS}

\section{Comparison of standard DDT and BMD method results}

Table 1 shows the CA rates between fluconazole susceptibility results obtained using the DDT and BMD methods for standard inoculum at 24 hours. The CA rates, determined using revised species-specific CBPs, between the two methods were 97.0\%, 98.6\%, 89.7\%, and 95.8\% for C. albicans, C. glabrata, C. parapsilosis, and $\mathrm{C}$. tropicalis, respectively. The overall CA rate for all 188 isolates was $96.3 \%$, with a VME rate of $1.6 \%$ and an ME rate of $2.1 \%$.

\section{DDT results after short incubation}

Table 3 shows the proportion (\%) of readable isolates (forming uniformly circular inhibition zones with sufficient growth) of the four Candida species in DDT using the two inocula (McFarland 0.5 or 2.5) and their CA with the BMD method according to the incubation time (10-24 hours). Of the 188 isolates, the propor- tions of readable DDT results obtained using the standard inoculum were $35.1 \%, 63.8 \%, 92.0 \%, 94.7 \%, 96.3 \%$, and $100 \%$ at $10,12,14,16,18$, and 22 hours, respectively. The proportions of readable DDT results obtained using the large inoculum were $58.5 \%, 91.0 \%, 96.8 \%$, and $100 \%$ at $10,12,14$, and 16 hours, respectively. Overall, DDT using the standard inoculum produced readable results for $>95 \%$ of $C$. albicans, $C$. glabrata, and $C$. tropicalis isolates at 14 hours, whereas results produced using the large inoculum were readable after 12 hours for $C$. glabrata and $C$. tropicalis and after 14 hours for $C$. albicans for $>95 \%$ of the isolates. For $C$. parapsilosis, the proportion of readable DDT results using the standard inoculum was $65.5 \%$ at 14 hours, which was significantly lower than that observed for the large inoculum $(89.7 \%, P<0.05)$. DDT results using the large inoculum were readable after 16 hours for $>95 \%$ of $C$. parapsilosis isolates, whereas the results using the standard inoculum were readable after 20 hours for $>95 \%$ of $C$. parapsilosis isolates.

Excellent CA (>90\%) between DDT and the BMD method for assessing fluconazole susceptibility was observed for each incubation time tested for nearly all C. albicans, C. glabrata, and C. tropicalis isolates, irrespective of the inoculum size. For C. parapsilosis, the CA rates for DDT using the standard inoculum were $68.4 \%, 89.7 \%$, and $89.7 \%$ at 14,20 , and 22 hours, whereas the CA rates for DDT using the large inoculum were $>92 \%$ at all time points, with no VMEs; at 14 hours, the CA rate for DDT

Table 4. Fluconazole DDT using two inocula at the first readable time and their categorical agreement with the BMD reference method for 188 isolates of four common Candida species*

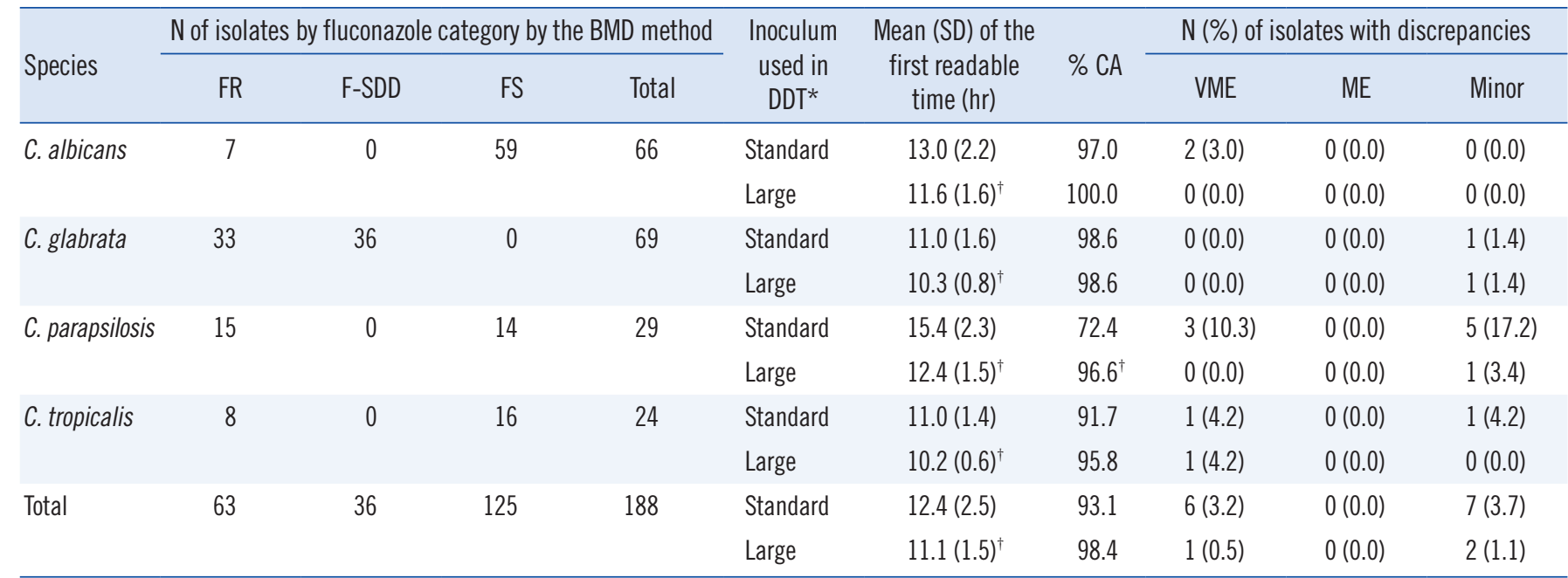

${ }^{*}$ CLSI M27 BMD method was conducted strictly according to CLSI M27-ED4 document (24-hour incubation), but DDT was modified with the use of two cell suspensions (McFarland 0.5, standard inoculum; and 2.5, large inoculum), and inhibition zones were read at 2-hour intervals from 10 hours to 22 hours [15]. ${ }^{\dagger} P<0.05$ between the standard inoculum and large inoculum within a given species.

Abbreviations: BMD, broth microdilution; CA, categorical agreement; DDT, disk diffusion testing; FR, fluconazole resistant; FS, fluconazole susceptible; FSDD, fluconazole-susceptible dose-dependent; ME, major error; Minor, minor error; SD, standard deviation; VME, very major error. 


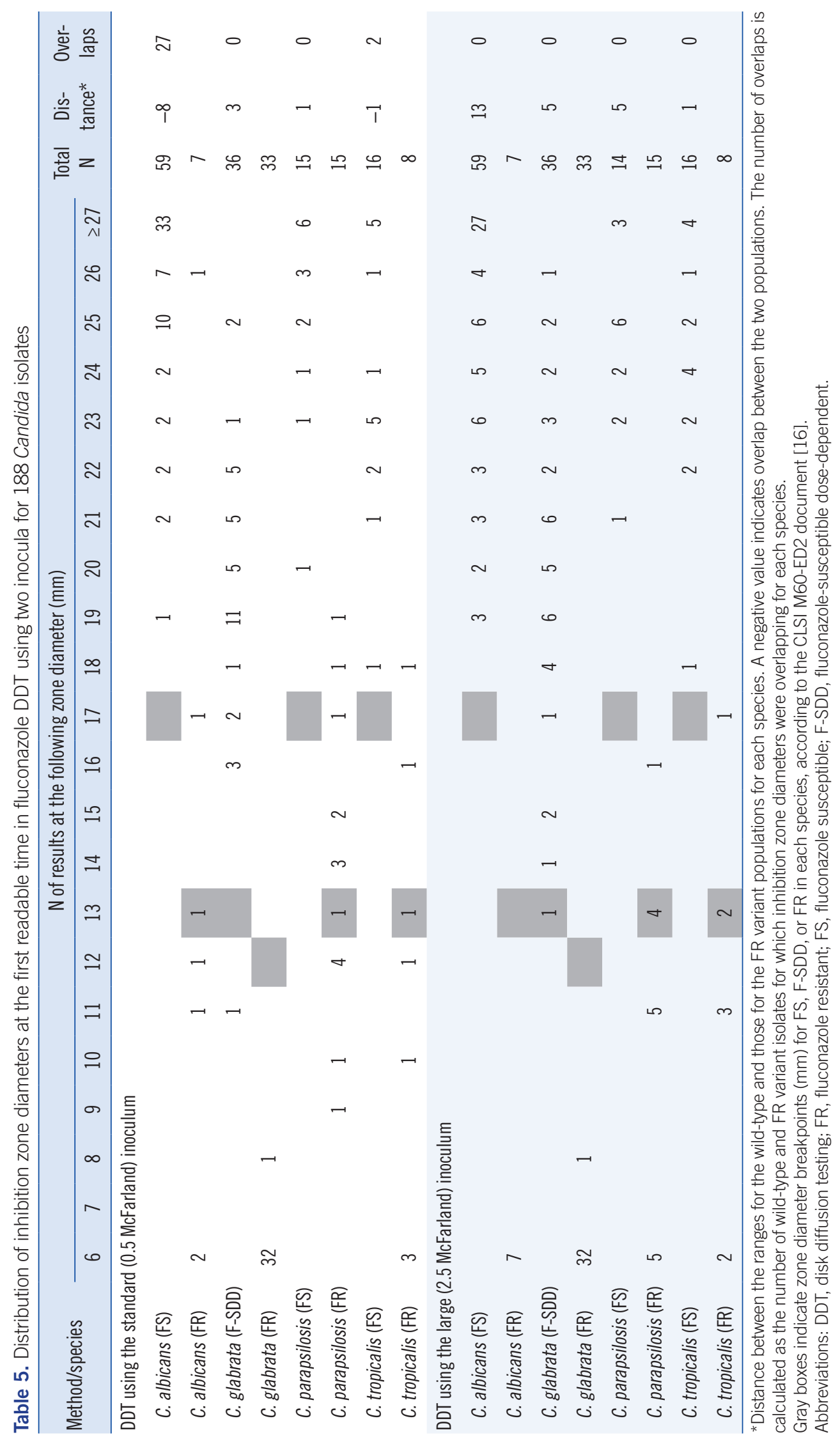


using the standard inoculum was significantly lower than that using the large inoculum $(68.4 \%$ vs. $92.3 \%, P<0.05)$.

\section{DDT results at first readable time}

Table 4 shows the average first readable times for DDT for all 188 Candida isolates and the CA with the BMD method. The first readable times were significantly shorter for the large inoculum than for the standard inoculum for each of the four Candida species separately and all four species combined (all, $P<0.05$ ). The CA rates for DDT using the standard or large inoculum over the BMD method were similar for $C$. albicans, $C$. glabrata, and C. tropicalis. However, the CA rate for $C$. parapsilosis was lower for DDT using the standard inoculum than for DDT using the large inoculum $(P<0.05)$. For the standard inoculum, three and five $C$. parapsilosis isolates with $\mathrm{Y} 132 \mathrm{~F}$ variants were misclassified as FS (VME rate, 10.3\%) and F-SDD (minor error rate, 17.2\%), respectively, at the first readable time.

Table 5 shows the distribution of DDT inhibition zone diameters obtained at the first readable time for the 188 Candida isolates using the standard or large inoculum. Wild-type and FR variant populations of $C$. albicans, C. glabrata, C. parapsilosis, and $C$. tropicalis were separated by $-8,3,2$, and $-1 \mathrm{~mm}$, respectively, in tests using the standard inoculum, and by $13,5,5$, and $1 \mathrm{~mm}$, respectively, in tests using the large inoculum. Overall, the large inoculum resulted in better separation between FS and FR variant isolates than the standard inoculum, and an overlap was found for $C$. albicans and $C$. tropicalis isolates in tests using the standard inoculum.

\section{DISCUSSION}

With an increase in FR among Candida isolates, rapid fluconazole susceptibility testing has become more important, particularly in patients with invasive candidiasis. We showed that DDT using a large inoculum (McFarland 2.5) enabled faster assessment of FR in four common Candida species, with virtually no VME results on FR categorization, than DDT using a standard inoculum (McFarland 0.5).

The standardized DDT (CLSI M44-ED1 or ED2), which uses McFarland 0.5 inoculum and a 24-hour incubation period, has been extensively validated in comparison with the CLSI M27 BMD reference method for the assessment of fluconazole sensitivity in common Candida species [8, 13, 14]. However, none of the previous studies relying on the standardized DDT provided species-specific results, as they were performed before the development of revised species-specific CBPs for DDT and the
CLSI M27 BMD method in 2010 [16].

In a large study of FR in 1,586 Candida isolates, the authors reported $93 \%$ CA, with VME and ME rates of only $0.1 \%$ and $0.3 \%$, respectively [14]. The low VME rate $(0.1 \%)$ in the previous study may be because FR isolates were rare in their collection (only $2.5 \%$ of isolates were FR) and partly because they used the previous CBP for the CLSI BMD method (MIC $\geq 64 \mu \mathrm{g} /$ $\mathrm{mL}$ for all Candida species) [19]. In our study testing well-characterized isolates from a Korean collection, in which 33.5\% of isolates were FR, the overall CA rate between the CLSI BMD method and DDT for all 188 isolates was 96.3\%, with VME and ME rates of $1.6 \%$ and $2.1 \%$, respectively. The present study revealed excellent CA between DDT (24-hour incubation) and the reference CLSI M27 BMD method using species-specific CBPs for fluconazole against C. albicans (97.0\%), C. glabrata (98.6\%), and $C$. tropicalis (95.8\%); however, the CA for molecularly defined isolates of $C$. parapsilosis in the Korean collection was lower (89.7\%).

To date, only three studies have directly evaluated DDT use for assessing fluconazole susceptibility in Candida species isolated from positive blood cultures [10, 11, 24]. These studies reported excellent CA between conventional (using colonies subcultured from positive blood cultures) and direct (using blood culture broth) DDT of fluconazole susceptibility [10, 11]. However, there were several potential shortcomings, including low numbers of antifungal-resistant isolates, the absence of earlier readings for DDT, and the lack of a direct comparison between DDT and the CLSI M27 BMD reference method. In the present study, Candida cell numbers in McFarland 0.5 and 2.5 inocula were $1.5-8.3 \times 10^{6} \mathrm{CFU} / \mathrm{mL}$ and $0.9-3.9 \times 10^{7} \mathrm{CFU} / \mathrm{mL}$, respectively, with considerable variation among the four Candida species. Given that Candida cell numbers in positive blood cultures range from $10^{5}$ to $10^{8} \mathrm{CFU} / \mathrm{mL}$, with most samples (87\%) being in the range $10^{6}-10^{7} \mathrm{CFU} / \mathrm{mL}$, we performed DDT using two cell suspensions, McFarland 0.5 (standard inoculum) and 2.5 (large inoculum) [11, 25].

DDT results were readable after 14 hours (C. albicans, C. glabrata, and C. tropicalis) and 20 hours (C. parapsilosis) for $>95 \%$ of the isolates when the standard inoculum was used, compared with 12 hours (C. glabrata and C. tropicalis), 14 hours (C. albicans), and 16 hours ( $C$. parapsilosis) when the large inoculum was used. Differences in the average first readable time between tests for DDT using standard and large inocula for $C$. albicans (13.0 hours vs. 11.6 hours), C. glabrata (11.0 hours vs. 10.3 hours), C. parapsilosis (15.4 hours vs. 12.4 hours), and C. tropicalis (11.0 hours vs. 10.2 hours) showed that DDT using large in- 
oculum may save up to 1-3 hours compared with the traditional method. Thus, DDT using the large inoculum may enable rapid detection of FR isolates of all four common Candida species.

We found that DDT using both standard and large inocula produced readable results after 14 hours of incubation for $>95 \%$ of C. albicans, C. glabrata, and C. tropicalis isolates. When the first readable DDT results were compared with those produced using the CLSI M27 BMD method, the CA rates was 97.0\%, 98.6\%, and 91.7\% for C. albicans, C. glabrata, and C. tropicalis, respectively, when the standard inoculum was used and $100 \%, 98.6 \%$, and $95.8 \%$, respectively, when the large inoculum was used. Overall, the CA between the methods was excellent (>90\%) for all three species across all time points (14-22 hours). However, VMEs were found for two (3\%) C. albicans isolates in DDT using the standard inoculum at the first readable time, while the U.S. Food and Drug Administration requires $<1.5 \%$ of resistant isolates evaluated to yield VMEs [26]. One FR isolate of $C$. albicans with a G464S variant in Erg11p with a fluconazole MIC of $8 \mathrm{~g} / \mathrm{mL}$ was consistently associated with VME (inhibition zone diameter, 17.25-18.27 mm, reading time, 14-24 hours) in DDT using the standard inoculum, but showed a decrease in inhibition zone diameter (6.0-17.3 mm) in DDT using the large inoculum, in which most VMEs changed into minor errors. The other FR isolate of $C$. albicans with a K143R variant in Erg11p and a fluconazole MIC of $8 \mathrm{~g} / \mathrm{mL}$ yielded a VME in DDT using both standard and large inocula, which may be attributed to the poor growth of the isolate on Mueller-Hinton agar. In addition, of the $15 \mathrm{FR}$ isolates of $C$. parapsilosis harboring the Y132F variant in Erg11p, three isolates with a fluconazole MIC of $8 \mathrm{~g} / \mathrm{mL}$ yielded a VME in DDT using the standard inoculum at an earlier reading time, although all three isolates exhibited a decrease in inhibition zone diameter after longer incubation. This is likely due to the relatively slow growth of $C$. parapsilosis on RPMI-glucose medium, suggesting that earlier reading of DDT results when the standard inoculum is used is unlikely during direct antifungal susceptibility testing of $C$. parapsilosis for fluconazole using DDT. Taken together, these results suggest that DDT using the large inoculum can be successfully used to test fluconazole susceptibility rapidly, which may lead to more appropriately targeted antifungal drug therapy and better outcomes in patients with candidemia due to common Candida species, especially $C$. parapsilosis.

When inhibition zone diameters at the first reading for each isolate were recorded, DDT using the standard inoculum showed overlap between wild-type isolates and FR variant populations for both $C$. albicans and C. tropicalis. However, a clear separa- tion between wild-type and FR variant isolates was observed for all isolates of $C$. glabrata (standard inoculum, $3 \mathrm{~mm}$; large inoculum, $5 \mathrm{~mm}$ ), and C. parapsilosis (standard inoculum, $1 \mathrm{~mm}$; large inoculum, $5 \mathrm{~mm}$ ) in DDT using both standard and large inocula, suggesting that DDT may be a promising tool for the rapid differentiation of $\mathrm{FR}$ variants from wild-type isolates of $C$. glabrata and C. parapsilosis, irrespective of the inoculum size. However, further studies using more isolates are needed to establish new breakpoints for rapid DDT for each Candida species by considering equivalent MIC values, pharmacokinetic and pharmacodynamic parameters, resistance mechanisms, and clinical outcomes, as these parameters relate to the DDT breakpoint values.

\section{ACKNOWLEDGEMENTS}

None.

\section{AUTHOR CONTRIBUTIONS}

Shin JH designed the study; Jeon S performed laboratory measurements; Lim HJ, Choi MJ, Byun SA, and Lee D collected and identified clinical isolates, and performed molecular studies; Shin $\mathrm{JH}$, Won EJ; and Jeon S wrote the preliminary manuscript; Shin $\mathrm{JH}$, Jeon S, and Lim HJ analyzed the data; Shin JH revised the manuscript; Lee SY, Won EJ, Kim SH, and Shin MG provided valuable comments and recommendations. All authors revised and accepted the final version of the manuscript.

\section{CONFLICTS OF INTEREST}

No potential conflicts of interest relevant to this article were reported.

\section{RESEARCH FUNDING}

This research was supported by the Basic Science Research Program through the National Research Foundation of Korea (NRF) funded by the Ministry of Education (NRF-2019R1A2 C1004644).

\section{ORCID}

Suhak Jeon https://orcid.org/0000-0002-9129-132X Jong Hee Shin https://orcid.org/0000-0001-9593-476X Ha Jin Lim https://orcid.org/0000-0003-2436-5007 
Min Ji Choi

https://orcid.org/0000-0001-8576-4015

Seung A Byun

https://orcid.org/0000-0003-4013-7274

Dain Lee

Seung Yeob Lee

Eun Jeong Won

Soo Hyun Kim

Myung Geun Shin

https://orcid.org/0000-0003-0236-9808

https://orcid.org/0000-0003-2851-1112

https://orcid.org/0000-0002-8750-4257

https://orcid.org/0000-0001-9739-711X

https://orcid.org/0000-0002-0372-9185

\section{REFERENCES}

1. Kullberg BJ and Arendrup MC. Invasive candidiasis. N Engl J Med 2015; 373:1445-56.

2. Lim HJ, Lee OJ, Kim SH, Shin MG, Shin JH. First Case of Nosocomial Fungemia Caused by Candida melibiosica. Ann Lab Med 2020;40:177179.

3. Pfaller MA, Diekema DJ, Turnidge JD, Castanheira M, Jones RN. Twenty years of the SENTRY antifungal surveillance program: results for Candida species from 1997-2016. Open Forum Infect Dis 2019;6(S1):S79-94.

4. Arendrup MC and Patterson TF. Multidrug-resistant Candida: epidemiology, molecular mechanisms, and treatment. J Infect Dis 2017;216(S3): S445-51.

5. Pappas PG, Kauffman CA, Andes DR, Clancy CJ, Marr KA, OstroskyZeichner $\mathrm{L}$, et al. Clinical practice guideline for the management of candidiasis: 2016 update by the Infectious Diseases Society of America. Clin Infect Dis 2016;62:e1-50.

6. Castanheira M, Deshpande LM, Davis AP, Rhomberg PR, Pfaller MA. Monitoring antifungal resistance in a global collection of invasive yeasts and molds: application of CLSI epidemiological cutoff values and wholegenome sequencing analysis for detection of azole resistance in Candida albicans. Antimicrob Agents Chemother 2017;61:e00906-17.

7. Choi YJ, Kim YJ, Yong D, Byun JH, Kim TS, Chang YS, et al. Fluconazole-resistant Candida parapsilosis bloodstream isolates with Y132F mutation in ERG11 gene, South Korea. Emerg Infect Dis 2018;24:176870.

8. Berkow EL, Lockhart SR, Ostrosky-Zeichner L. Antifungal susceptibility testing: current approaches. Clin Microbiol Rev 2020;33: e00069-19.

9. van den Bijllaardt W, Buiting AG, Mouton JW, Muller AE. Shortening the incubation time for antimicrobial susceptibility testing by disk diffusion for Enterobacteriaceae: how short can it be and are the results accurate? Int J Antimicrob Agents 2017;49:631-7.

10. Jabeen K, Kumar H, Farooqi J, Mehboob R, Brandt ME, Zafar A. Agreement of direct antifungal susceptibility testing from positive blood culture bottles with the conventional method for Candida species. J Clin Microbiol 2016;54:343-8.

11. Oz $\mathrm{Y}$ and Gokbolat E. Evaluation of direct antifungal susceptibility testing methods of Candida spp. from positive blood culture bottles. J Clin Lab Anal 2018;32:e22297.

12. Jonasson E, Matuschek E, Kahlmeter G. The EUCAST rapid disc diffusion method for antimicrobial susceptibility testing directly from positive blood culture bottles. J Antimicrob Chemother 2020;75:968-78.

13. Meis J, Petrou M, Bille J, Ellis D, Gibbs D. A global evaluation of the susceptibility of Candida species to fluconazole by disk diffusion. Global Antifungal Surveillance Group. Diagn Microbiol Infect Dis 2000;36:215-
23.

14. Pfaller MA, Diekema DJ, Messer SA, Boyken L, Hollis RJ. Activities of fluconazole and voriconazole against 1,586 recent clinical isolates of Candida species determined by broth microdilution, disk diffusion, and Etest methods: report from the ARTEMIS Global Antifungal Susceptibility Program, 2001. J Clin Microbiol 2003;41:1440-6.

15. CLSI. Reference method for broth dilution antifungal susceptibility testing of yeasts. 4th ed. (M27-Ed4). Wayne, PA: Clinical and Laboratory Standards Institute, 2017.

16. CLSI. Performance standards for antifungal susceptibility testing of yeasts. 2nd ed. (M60-Ed2). Wayne, PA: Clinical and Laboratory Standards Institute, 2020.

17. Lee HS, Shin JH, Choi MJ, Won EJ, Kee SJ, Kim SH, et al. Comparison of the Bruker Biotyper and VITEK MS matrix-assisted laser desorption/ ionization time-of-flight mass spectrometry systems using a formic acid extraction method to identify common and uncommon yeast isolates. Ann Lab Med 2017;37:223-230.

18. Byun SA, Won EJ, Kim MN, Lee WG, Lee K, Lee HS, et al. Multilocus sequence typing (MLST) genotypes of Candida glabrata bloodstream isolates in Korea: association with antifungal resistance, mutations in mismatch repair gene (Msh2), and clinical outcomes. Front Microbiol 2018;9:1523.

19. Lim HJ, Shin JH, Kim MN, Yong D, Byun SA, Choi MJ, et al. Evaluation of two commercial broth microdilution methods using different interpretive criteria for the detection of molecular mechanisms of acquired azole and echinocandin resistance in four common Candida species. Antimicrob Agents Chemother 2020;64:e00740-20.

20. Morio F, Pagniez F, Besse M, Gay-andrieu F, Miegeville M, Le Pape P. Deciphering azole resistance mechanisms with a focus on transcription factor-encoding genes TAC1, MRR1 and UPC2 in a set of fluconazoleresistant clinical isolates of Candida albicans. Int J Antimicrob Agents 2013;42:410-5.

21. Arastehfar A, Daneshnia F, Zomorodian K, Najafzadeh MJ, Khodavaisy $\mathrm{S}$, Zarrinfar $\mathrm{H}$, et al. Low level of antifungal resistance in Iranian isolates of Candida glabrata recovered from blood samples in a multicenter study from 2015 to 2018 and potential prognostic values of genotyping and sequencing of PDR1. Antimicrob Agents Chemother 2019;63:e0250318.

22. Choi MJ, Won EJ, Shin JH, Kim SH, Lee WG, Kim MN, et al. Resistance mechanisms and clinical features of fluconazole-nonsusceptible Candida tropicalis isolates compared with fluconazole-less-susceptible isolates. Antimicrob Agents Chemother 2016;60:3653-61.

23. CLSI. Method for antifungal disk diffusion susceptibility testing of yeasts. 3rd ed. (M44-Ed3). Wayne, PA: Clinical and Laboratory Standards Institute, 2018

24. Kumar H, Farooqi J, Mehboob R, Jabeen K, Zafar A. Optimization of protocol for disc diffusion of antifungal drugs directly from blood culture bottles positive for Candida species. Infect Dis J Pak 2015;24:809-12.

25. Chang HC, Chang JJ, Chan SH, Huang AH, Wu TL, Lin MC, et al. Evaluation of Etest for direct antifungal susceptibility testing of yeasts in positive blood cultures. J Clin Microbiol 2001;39:1328-33.

26. Humphries RM, Ambler J, Mitchell SL, Castanheira M, Dingle T, Hindler JA, et al. CLSI methods development and standardization working group best practices for evaluation of antimicrobial susceptibility tests. J Clin Microbiol 2018;56:e01934-17. 